\title{
Diuréticos y betabloqueantes previenen la insuficiencia cardíaca en pacientes mayores con hipertensión sistólica aislada
}

Prevention of Heart Failure by Antihypertensive Drug Treatment in Older Persosns With Isolated Systolic Hypertension.

The SHEP Cooperative Reserch Group. JAMA 1997; 278:212-216

\section{Objetivo}

Determinar el efecto del tratamiento de la Hipertensión Sistólica Aislada (HTSA) en el desarrollo de Insuficiencia Cardíaca (IC) en mayores de 60 años; con un esquema basado en diuréticos.

Lugar

Varios centros de EEUU.

\section{Pacientes}

Se incluyeron 4736 pacientes (57\% mujeres) mayores de 60 años (media=72) con tensión arterial sistólica (TAS) entre 160 y 220 $\mathrm{mmHg}$ y TA diastólica $<90 \mathrm{mmHg}$. Los pacientes con antecedentes de infarto de miocardio (IAM) fueron analizados como subgrupo.

\begin{abstract}
Intervención
Tratamiento escalonado de La HTSA con un esquema a base de diuréticos. Se definió como control de la HTSA al descenso de la TAS de $21 \mathrm{mmHg}$ o TAS $<160 \mathrm{mmHg}$ ):

1er paso: Clortalidona $12,5 \mathrm{mg} /$ día y aumento a $25 \mathrm{mg} /$ día si no se logra control

2do paso: Agregado de atenolol $25 \mathrm{mg} /$ día (reserpina si estaba contraindicado) y aumento a $50 \mathrm{mg} /$ día si no se logra control.

Si no se alcanzaba el control se informaba el régimen que el paciente recibía y se los trataba abiertamente.
\end{abstract}

Medición de resultados principales

Insuficiencia cardíaca fatal y no fatal (definición clínico-radiológica)

Resultados Principales

El análisis fue por intención de tratar* .

\begin{tabular}{llllll}
\hline Eventos de IC & $\begin{array}{l}\text { Placebo } \\
\mathrm{n}(\%)\end{array}$ & $\begin{array}{l}\text { Tto. activo } \\
\mathrm{n}(\%)\end{array}$ & $\begin{array}{l}\text { Riesgo Relativo } \\
\text { RR (IC 95\%) }\end{array}$ & $\mathrm{p}$ & NNT \\
\hline Número de pacientes & 2371 & 2365 & - & - & - \\
\hline IC no fatal & $102(4.3)$ & $48(2.0)$ & $0.46(0.33-0.65)$ & $<0.001$ & 44 \\
\hline Internación por IC no fatal & $75(3.2)$ & $38(1.6)$ & $0.50(0.34-0.74)$ & $<0.001$ & 65 \\
\hline IC fatal + no fatal & $105(4.4)$ & $55(2.3)$ & $0.51(0.37-0.71$ & $<0.001$ & 48 \\
\hline IC fatal + Internac. por IC no fatal & $79(3.3)$ & $45(1.9)$ & $0.57(0.34-0.81)$ & 0.002 & 70 \\
\hline Mort. Cardíaca + Internac. IC no fatal & $162(6.8)$ & $113(4.8)$ & $0.69(0.54-0.87)$ & 0.002 & 49 \\
\hline Mort. Cardiovascular + Internac. IC no fatal & $174(7.3)$ & $123(5.2)$ & $0.70(0.55-0.88)$ & 0.002 & 47 \\
\hline
\end{tabular}

NNT: número necesario para tratar durante 4.5 años para prevenir un evento *

Al 44,4\% de los pacientes del grupo placebo se los trató abiertamente debido a la falta de control. En el subgrupo de pacientes con IAM previo el beneficio fue considerablemente mayor: RR para desarrollo de IC $0.19(0.06-0.53)$ y un NNT de 15.

\section{Conclusión}

El tratamiento de la HTSA en pacientes mayores de 60 años disminuye a la mitad el riesgo de desarrollar IC en los 4,5 años subsiguientes. El beneficio es mayor en aquellos pacientes con antecedentes de IAM.

\section{COMENTARIO}

Hace más de seis años el estudio SHEP (Systolic Hipertension Elderly Program) demostró el beneficio de tratar la HTSA en mayores de 60 años para prevenir accidentes cerebro-vasculares y eventos cardiovasculares totales (1). En el presente trabajo se evalúa la incidencia de IC, utilizando la misma base de datos. La magnitud del estudio (más de 4700 participantes) les permite a los autores hacer este nuevo análisis de un resultado que en la publicación original fue considerado como secundario. Es muy útil observar la forma de presentación de los resultados, no sólo con los riesgos relativos de padecer cada evento, sino con el número necesario para tratar (NNT) que permite apreciar fácilmente la importancia de la intervención en el grupo de pacientes. En este caso, tratando 48 pacientes con HTSA durante 4,5 años se puede prevenir la aparición de una IC. Además los autores consideran varios eventos aislados o combinados y el beneficio del tratamiento es consistente en todas las categorías. Si bien el estudio considera el diagnóstico de IC a través del interrogatorio, examen físico, y radiografía de tórax; estos elementos le dan al estudio mayor validez externa en nuestro medio, donde no utilizamos rutinariamente otros estudios complementarios para el diagnostico de esta entidad clínica. Hay que recalcar también que a pesar de la aparición de nuevas técnicas como la presurometría ambulatoria, la evidencia apoyada por este y otros estudios avala utilizar esfigmomanómetros manuales para el seguimiento de pacientes hipertensos. El alto porcentaje de pacientes tratados abiertamente en el grupo placebo $(44,4 \%)$ al final del estudio sugiere que el beneficio de la intervención es probablemente mayor que el observado, ya que aún habiendo recibido tratamiento activo, estos pacientes son considerados como pertenecientes al grupo placebo en el análisis (intención de tratar). Este estudio no hace más que reforzar la indicación de diuréticos y betabloqueantes en el tratamiento inicial de la hipertensión arterial.

*Ver glosario

\section{Dr. Juan Pablo Roubicek}

Servicio de Internación Domiciliaria Hospital Privado de Comunidad de Mar del Plata 